\title{
Produtividade do feijoeiro comum influenciada por plantas de cobertura e sistemas de manejo do solo
}

\author{
Enderson P. de B. Ferreira $^{1}$, Luís F. Stone ${ }^{1}$, Fábio L. Partelli² \& Agostinho D. D idonet ${ }^{1}$
}

\begin{abstract}
RESUMO
Conduziu-se um experimento de campo sob sistema plantio direto (SPD) e sistema de plantio convencional (SPC) para avaliar a produção de massa seca, a quantidade de $\mathrm{N}$ e a taxa de decomposição de crotalária (Crotalaria juncea) e sorgo forrageiro (Sorghum bicolor L.), e seus efeitos na produtividade do feijoeiro comum (Phaseolus vulgaris L.). O sistema de manejo do solo não influenciou na produtividade de massa seca das plantas de cobertura do solo; contudo, o sorgo produziu $60 \%$ mais massa seca que a crotalária. 0 maior acúmulo de N foi observado sob SPD, 45\% a mais que sob SPC; entretanto, não houve diferença entre as plantas de cobertura do solo. A taxa de decomposição da palhada sob SPD foi $50 \%$ menor do que sob SPC e, até os 28 dias, a decomposição da crotalária sob SPC foi mais rápida que a do sorgo. A produtividade de grãos da cultura do feijoeiro comum foi $40 \%$ maior em SPD em comparação com o SPC apresentando alta correlação com a quantidade de $\mathrm{N}$ acumulada pelas plantas de cobertura do solo. Sob as condições do Cerrado Goiano, o SPD promove maior acúmulo de $\mathrm{N}$ e menor taxa de decomposição das plantas de cobertura do solo, promovendo a maior produtividade da cultura do feijoeiro comum, comparado ao SPC.
\end{abstract}

Palavras-chave: crotalária, sorgo, palhada, taxa de decomposição

\section{Grain yield of common bean as affected by cover crops and soil management systems}

\begin{abstract}
A field experiment was carried out under no-tillage system (NT) and conventional tillage (CT) to assess the dry mass production, $\mathrm{N}$ accumulation and the decomposition rate of crotalaria (Crotalaria juncea) and sorghum (Sorghum bicolor L.), as well as, their effects on the grain yield of common bean (Phaseolus vulgaris L.). Results showed that soil management had no effect on the dry mass production of cover crop, however, the sorghum produced $60 \%$ more dry mass as compared with crotalaria. The largest $\mathrm{N}$ accumulation was observed under NT, which showed $45 \%$ more $\mathrm{N}$ than $\mathrm{CT}$, however, no statistical difference between cover crops was observed. The straw rate decomposition under NT was $50 \%$ lower than under CT and, until 28 days, the decomposition of crotalaria under CT was faster than sorghum. The common bean grain yield was $40 \%$ higher under NT as compared with CT and it showed high correlation with $\mathrm{N}$ amount accumulated by the cover crops. Under Cerrado condition of Goiás state, NT promotes greater $\mathrm{N}$ accumulation and lower decomposition rate of the cover crops, leading to greater grain yield of common bean as compared with $\mathrm{CT}$.
\end{abstract}

Key words: crotalaria, sorghum, straw, decomposition rate

\footnotetext{
1 Embrapa Arroz e Feijão, C.P. 179, CEP.75375-000, Santo Antônio de Goiás, G O. Fone: (62) 3533-2151. E-mail: enderson@cnpaf.embrapa.br; stone@cnpaf.embrapa.br; didonet@cnpaf.embrapa.br

2 U niversidade Federal do Espírito Santo, Departamento de Ciências Agrárias e Biológicas - DCAB/U FES, Rodovia BR 101 N orte, Km. 60 , Bairro Litorâneo, CEP 29932-540, São Mateus, ES. Fone: (27) 3312-1511. E-mail: partelli@yahoo.com.br
} 


\section{INTRODUÇÃO}

A agricultura convencional trouxe muito progresso para a Ciência Agronômica em termos de conhecimentos e de produtividade por unidade de área em curto prazo; entretanto, o uso inadequado dessa tecnologia tem ocasionado degradação do solo e do ambiente (Carneiro et al., 2004). Ademais, ela requer maior consumo de energia fóssil, principalmente pelo alto consumo de fertilizantes e defensivos agrícolas (Castellini et al., 2006), e contribui para o efeito estufa através do aumento da liberação de $\mathrm{CO}_{2}$ para a atmosfera (Kaltsas et al., 2007), colocando em cheque a sobrevivência desse modelo de desenvolvimento.

Os sistemas de produção agroecológicos têm sido utilizados como estratégia para a produção de alimentos visando à preservação ambiental e à biodiversidade, sem provocar danos à saúde (Welch \& Graham, 1999), uma vez que preconizam a diversificação de cultivos, o manejo ecológico do solo e o controle biológico de pragas, considerando a estabilidade ecológica, a equidade social e a aceitabilidade cultural dos estilos de agricultura a serem implementados (Altieri, 1994). Portanto, a agricultura sustentável, produtiva e ambientalmente equilibrada, apoia-se em práticas conservacionistas de preparo do solo, rotação de culturas e consórcios, no uso de adubos verdes e de controle biológico de pragas, tal como no emprego eficiente dos recursos naturais. Infere-se, daí, que os processos biológicos que ocorrem no sistema solo-planta, efetivados por micro-organismos e pequenos invertebrados, constituem a base sobre a qual a agricultura agroecológica se sustenta (Faria \& Franco, 2002).

Neste contexto, o grande desafio na transição de um sistema produtivo convencional para um sistema sustentável está na recuperação da estrutura física, química, biológica e da fertilidade do solo, o qual, em muitas situações, encontra-se degradado, com queda de produtividade mesmo com altíssimo uso de insumos externos (Altieri, 1999). Para tanto, a adoção de medidas conservacionistas, dentre elas a utilização de plantas de cobertura, desponta como prática viável, pelo fato de favorecer a densidade e diversidade de micro-organismos edáficos (Carneiro et al., 2004), melhorar a estrutura do solo (Carvalho et al., 2004; Andrade et al., 2009; Cunha et al., 2010), proporcionar a ciclagem de nutrientes e, quando se utilizam leguminosas, também promover, juntamente com bactérias específicas, a fixação biológica do nitrogênio atmosférico e o consequente aumento da produtividade das culturas (Chu et al., 2004; Teixeira et al., 2006; Pietsch et al., 2007).

Plantas de cobertura do solo, como crotalária (Crotalaria juncea) e sorgo forrageiro (Sorghum bicolor L.), apresentam diferentes relações $\mathrm{C} / \mathrm{N}$, determinante para o tempo de decomposição do material vegetal deixado na superfície ou incorporado ao solo e, consequentemente, para a disponibilização dos nutrientes às plantas. Este processo de transformação da matéria orgânica é realizado primariamente pelos micro-organismos do solo (Waksman et al., 1928), os quais desempenham importante papel no fluxo de energia e ciclagem de nutrientes e são considerados importantes indicadores da qualidade do solo (Ferreira et al., 2010).
A deposição de material vegetal na superfície do solo, comparada com sua incorporação, apresenta várias vantagens, como: proteção do solo, retenção de umidade, controle de plantas espontâneas, liberação mais lenta de nutrientes, maior atividade enzimática, teor de $\mathrm{C}$ orgânico, $\mathrm{C}$ e $\mathrm{N}$ da biomassa microbiana e estabilidade de agregados (Landers, 2001; Roldán et al., 2003; 2007; Roscoe et al., 2006); entretanto, esta disponibilização de nutrientes no solo deve ocorrer no momento de maior demanda da planta, o que varia em função da espécie cultivada e da forma de manejo da palhada e do solo.

O feijoeiro comum (Phaseolus vulgaris L.) é uma cultura de extrema importância para a sociedade brasileira, uma vez que é uma das fontes de proteína mais utilizada pela população mais carente (Paula Júnior et al., 2008). Cultivares melhoradas de feijoeiro comum com capacidade de expressão de alta produtividade, ampla adaptação, menor sensibilidade aos estresses bióticos ou abióticos e ciclo variando de 75 a 98 dias, representam uma das mais significativas contribuições à eficiência do setor produtivo (Silva \& Del Peloso, 2006).

Devido a todas essas características, a escolha do manejo adequado das plantas de cobertura e do solo é fator primordial para o sucesso do uso dessas plantas como fontes de nutrientes para a cultura do feijoeiro comum. O objetivo deste trabalho foi avaliar a produção de massa seca, a quantidade de $\mathrm{N}$ e a taxa de decomposição da crotalária e do sorgo forrageiro, associadas a sistemas de manejo do solo e seus efeitos na produtividade do feijoeiro comum.

\section{Material e MÉTOdos}

O experimento foi conduzido na Estação Experimental em Agroecologia da Embrapa Arroz e Feijão, em Santo Antônio de Goiás, GO, cujas coordenadas geográficas são: latitude $16^{\circ} 29^{\prime}$ $40^{\prime \prime} \mathrm{S}$, longitude $49^{\circ} 17^{\prime} 30^{\prime \prime} \mathrm{W}$ e altitude de $823 \mathrm{~m}$. De acordo com a classificação de Köppen, o clima do local é classificado como Aw, tropical de savana, megatérmico. O regime de chuvas é bem definido, com uma estação chuvosa de outubro a março e uma estação seca de abril a setembro, com pluviosidade anual média de $1460 \mathrm{~mm}$ (Silva et al., 2002).

O solo do local é um Latossolo Vermelho distrófico com as seguintes características: areia $=410 \mathrm{~g} \mathrm{~kg}^{-1}$, silte $=270 \mathrm{~g} \mathrm{~kg}^{-1} \mathrm{e}$ argila $=320 \mathrm{~g} \mathrm{~kg}^{-1}$; matéria orgânica $=2,01 \%, \mathrm{P}=5,8 \mathrm{mg} \mathrm{dm}^{-3}, \mathrm{~K}$ $=145,9 \mathrm{mg} \mathrm{dm}^{-3}, \mathrm{Ca}=3,6 \mathrm{cmol}_{\mathrm{c}} \mathrm{dm}^{-3}, \mathrm{Mg}=1,2 \mathrm{cmol}_{\mathrm{c}} \mathrm{dm}^{-3} \mathrm{e} \mathrm{pH}$ em água $(1: 2.5)=6,2$.

A condução do experimento ocorreu de outubro de 2008 a março de 2009, em plena estação chuvosa. Os dados climáticos do período, medidos na estação meteorológica da Embrapa Arroz e Feijão, são apresentados na Tabela 1.

O delineamento experimental foi o de blocos ao acaso, no esquema de parcelas subdivididas, com três repetições. Nas parcelas foram dispostos dois manejos de solo, sistema plantio direto (SPD) e sistema de plantio convencional (SPC) com grades aradora e niveladora operando até $10-15 \mathrm{~cm}$ de profundidade e nas subparcelas duas plantas de cobertura do solo, crotalária e o sorgo forrageiro. 
Tabela 1. Caracterização climática na área experimental durante a condução do ensaio

\begin{tabular}{|c|c|c|c|c|c|c|}
\hline & \multicolumn{2}{|c|}{ Temperatura média ( ${ }^{\circ} \mathrm{C}$ ) } & \multirow{2}{*}{$\begin{array}{c}\text { Umidade do ar } \\
(\%)\end{array}$} & \multirow{2}{*}{$\begin{array}{l}\text { Precipitação total } \\
(\mathrm{mm})\end{array}$} & \multirow{2}{*}{$\begin{array}{l}\text { Evapotranspiração total } \\
(\mathrm{mm})\end{array}$} & \multirow{2}{*}{$\begin{array}{l}\text { Radiação solar } \\
\left(\mathrm{h} \mathrm{d}^{-1}\right)\end{array}$} \\
\hline & Máxima & Mínima & & & & \\
\hline \multicolumn{7}{|c|}{ Cultivo das coberturas do solo } \\
\hline Out/2008 & 32,6 & 19,8 & 58,3 & 122,0 & 199,2 & 8,0 \\
\hline Nov2008 & 31,0 & 19,7 & 71,2 & 219,8 & 138,5 & 5,7 \\
\hline Dez/2008 & 29,5 & 19,5 & 75,1 & 230,4 & 118,1 & 5,1 \\
\hline Média & 31,0 & 19,7 & 68,2 & 190,7 & 151,9 & 6,3 \\
\hline \multicolumn{7}{|c|}{ Cultivo do feijoeiro comum } \\
\hline an/2009 & 29,5 & 19,6 & 77,6 & 221,4 & 147,2 & 6,8 \\
\hline Fev/2009 & 29,5 & 19,3 & 79,0 & 161,9 & 136,3 & 6,3 \\
\hline Mar/2009 & 30,4 & 19,2 & 76,4 & 193,2 & 150,4 & 7,0 \\
\hline Média & 29,8 & 19,4 & 77,7 & 192,2 & 144,6 & 6,7 \\
\hline
\end{tabular}

Os manejos de solo foram implantados em janeiro de 2008 e as plantas de cobertura em outubro de 2008, em SPD, no espaçamento de $40 \mathrm{~cm}$ entre linhas com 35 e 15 sementes por metro de crotalária e sorgo, respectivamente. A cultivar BRS Horizonte de feijoeiro comum foi cultivada em segunda safra (janeiro de 2009), sob SPD e SPC, em espaçamento de $40 \mathrm{~cm}$ entre linhas com 15 sementes por metro. Tanto as parcelas das culturas de cobertura, quanto as parcelas de feijoeiro comum, tinham $12 \mathrm{~m}^{2}$ de área.

A coleta das plantas de cobertura do solo para determinação da produtividade de massa seca e percentual de $\mathrm{N}$ nos tecidos, foi realizada na fase de floração. Com vista à determinação da produtividade de massa seca foram coletadas três linhas de 1 metro por parcela. $\mathrm{O}$ material foi colocado para secar a $65^{\circ} \mathrm{C} \mathrm{em}$ estufa até atingir massa constante. Para a determinação do aporte de $\mathrm{N}$ ao sistema produtivo, após a secagem, as plantas inteiras (folhas e caules/colmos) foram moídas e, então, determinado o teor de $\mathrm{N}$ pelo método de Kjeldhal descrito por Tedesco et al. (1995).

A determinação da decomposição foi realizada após o manejo da crotalária e do sorgo pelo uso do equipamento denominado "triton". Coletou-se o material vegetal picado deixado sobre o solo, que foi colocado para secar em estufa, até atingir massa constante. Para esta avaliação foram confeccionadas bolsas de decomposição (litter bags) de $30 \times 15 \mathrm{~cm}$ com tela de polietileno de $1 \mathrm{~mm}$ de malha. Em cada bolsa de decomposição foram adicionados $10 \mathrm{~g}$ do material vegetal seco, o que equivale a aproximadamente $3,3 \mathrm{Mg}$ de massa seca ha- ${ }^{-1}$.

No SPD, as bolsas de decomposição foram deixadas sobre a superfície do solo enquanto no SPC foram enterradas a $20 \mathrm{~cm}$ de profundidade, simulando as condições de campo. Neste caso, as bolsas de decomposição foram enterradas usando-se duas formas de colocação: i) as bolsas de decomposição foram colocadas no fundo de uma cova com $20 \mathrm{~cm}$ de profundidade e cobertas com solo e ii) as bolsas de decomposição foram colocadas inclinadas, em um ângulo de $45^{\circ}$, de forma que ocupavam toda a profundidade de 0 a $20 \mathrm{~cm}$. Aos 4, 12, 28, $60 \mathrm{e}$ 124 dias após sua colocação (DAC), as bolsas de decomposição foram recuperadas e o material remanescente dentro delas foi colocado para secar em estufa, para a determinação da sua massa seca.

A avaliação da produtividade da cultura do feijoeiro comum foi realizada em uma área útil de 1,2 $\mathrm{m}^{2}$, coletada na região central de cada parcela. Após a coleta, os grãos foram trilhados e colocados para secar em estufa. A umidade dos grãos foi corrigida para $13 \%$ e a produtividade expressa em kg ha-1.

Os dados referentes à produção de massa seca e quantidade de $\mathrm{N}$ das coberturas do solo e de produtividade do feijoeiro comum, foram submetidos a análise de variância e as médias foram comparadas pelo teste de Tukey a 5\% de significância. Os valores referentes à quantidade de massa seca remanescente das coberturas do solo e à sua taxa de decomposição, foram submetidos à análise de regressão com o software SISVAR (Ferreira, 2003). Análises de correlação de Pearson foram realizadas utilizando-se o software $\mathrm{R}$ (Wessa, 2008) entre os dados de produtividade de grãos e quantidade de massa seca e de $\mathrm{N}$ fornecidas pelas plantas de cobertura do solo.

\section{RESULTADOS E DISCUSSÃO}

A produtividade de massa seca das plantas de cobertura do solo não foi influenciada significativamente pelos sistemas de manejo de solo(Figura 1A). A maior quantidade de massa seca foi produzida pelo sorgo, $9,56 \mathrm{Mg} \mathrm{ha}^{-1}$, enquanto a crotalária produziu em torno de $6 \mathrm{Mg} \mathrm{ha}^{-1}$. Em relação à crotalária, os resultados observados foram similares aos encontrados por Bordin et al. (2003); entretanto, para o sorgo esses mesmos autores relataram produção de massa seca $20 \%$ superior ao encontrado neste trabalho.

A quantidade de $\mathrm{N}$ acumulada pelas plantas de cobertura do solo sob SPD foi cerca de $45 \%$ maior do que sob SPC; entretanto, não foi observado efeito da espécie de planta de cobertura do solo, apesar do sorgo ter acumulado o equivalente

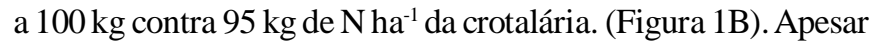
de não ter sido observada diferença significativa entre as plantas de cobertura do solo, neste trabalho elas forneceram $97,5 \mathrm{~kg}_{\text {de }} \mathrm{N} \mathrm{ha}^{-1}\left(95 \mathrm{~kg}\right.$ de $\mathrm{N} \mathrm{ha}^{-1}$ pela crotalária e $100 \mathrm{~kg}$ de $\mathrm{N}$ ha $^{-1}$ pelo sorgo), o que é suficiente para atender a grande parte da demanda de $\mathrm{N}$ da maioria das culturas anuais, como o feijoeiro comum. Segundo Santos et al. (2003), a cultura do feijoeiro comum atinge $90 \%$ da produtividade fisiológica $\left(2.478 \mathrm{~kg} \mathrm{ha}^{-1}\right)$ com $108 \mathrm{~kg}$ de $\mathrm{N} \mathrm{ha}^{-1}$. Barbosa Filho \& Silva (2000) relataram que o feijoeiro respondeu à adubação nitrogenada em cobertura, atingindo produção máxima de grãos, de $3.170 \mathrm{~kg} \mathrm{ha}^{-1} \mathrm{com}$ a aplicação de $120 \mathrm{~kg}_{\text {de }} \mathrm{N} \mathrm{ha}^{-1}$. Considerando ainda que o sorgo apenas recicla o $\mathrm{N}$ do solo, enquanto a crotalária obtém parte do N pelo processo de Fixação Biológica do Nitrogênio (FBN), fica evidente a grande importância dessa leguminosa para a manutenção da sustentabilidade do sistema produtivo. 

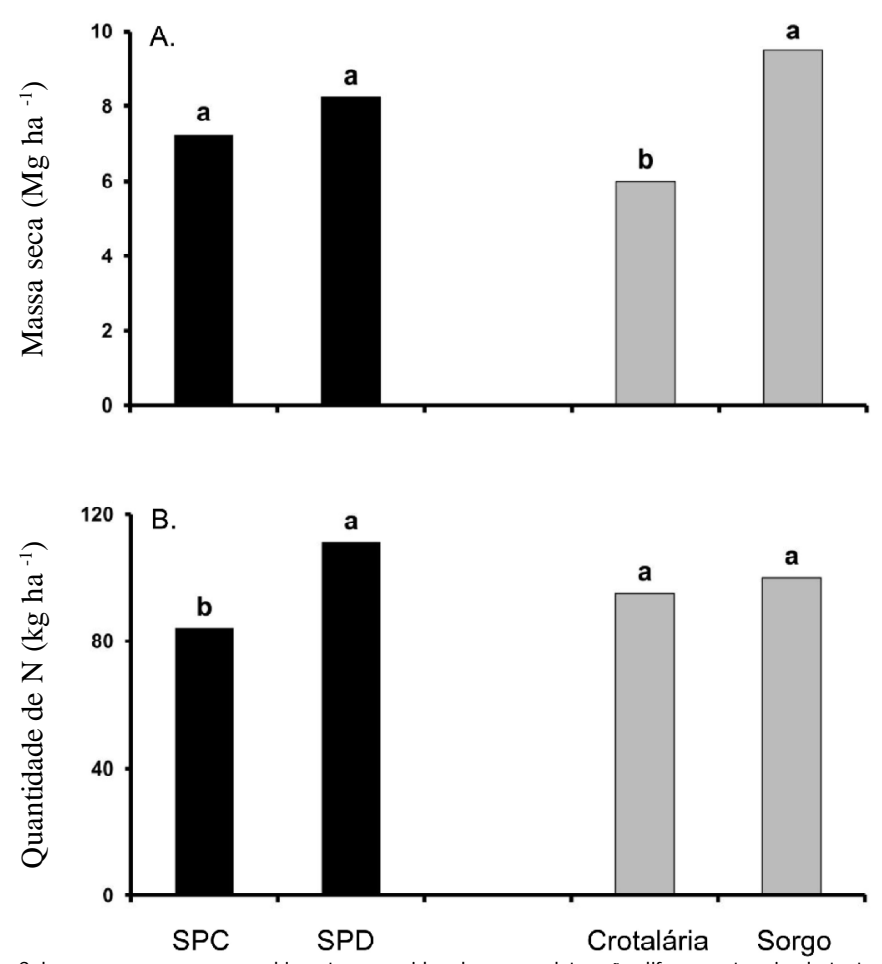

Colunas com mesmo preenchimento e seguidas da mesma letra não diferem entre si pelo teste de Tukey $(p<0,05)$

Figura 1. Produtividade de massa seca das plantas de cobertura do solo sob sistema plantio direto (SPD) e sistema de plantio convencional (SPC), e por espécie (A). $\mathrm{Q}$ uantidade de $\mathrm{N}$ acumulada pelas plantas de cobertura do solo sob sistema plantio direto (SPD) e sistema de plantio convencional (SPC), e por espécie (B)

Esses resultados corroboram com os relatados por Aguiar et al. (2010), que em um estudo com plantas de cobertura do solo, em um Latossolo Vermelho distrófico do Cerrado, reportaram diferença significativa entre crotalária e sorgo para a produção de massa seca; no entanto, sem diferença entre essas plantas para a quantidade de $\mathrm{N}$.

A avaliação da decomposição das palhadas de crotalária e de sorgo indicou que houve influência do tipo de sistema de manejo do solo e do tempo de amostragem, sendo mais lenta sob SPD (Tabela 2). Segundo Dalal et al. (2007), comparado ao SPC o SPD diminui a decomposição da palhada, aumentando a imobilização do $\mathrm{N}$ e o teor de carbono orgânico no solo.

Até os quatro DAC não foi observada qualquer diferença na taxa de decomposição entre as plantas de cobertura (Tabela
2). A partir dos 12 DAC foram observadas diferenças na decomposição das plantas de cobertura do solo em função do sistema de manejo, sendo a taxa de decomposição maior sob SPC. Diferenças nas taxas de decomposição da crotalária e do sorgo só foram observadas aos $28 \mathrm{DAC}$, sendo maior na crotalária. A forma de colocação das bolsas de decomposição, contudo, não influenciou a taxa de decomposição da palhada de crotalária, bem como a de sorgo (Tabela 2).

Observa-se que houve diferença significativa na taxa de decomposição de todos os tratamentos, em função da época de amostragem. Além disso, até os 60 DAE em SPD haviam sido decompostos cerca de $50 \%$ da massa seca $(52,95 \%$ para a crotalária e 47,43\% para o sorgo), enquanto sob SPC restava, no mesmo período, apenas cerca de $15 \%$ para a crotalária e $25 \%$ para o sorgo (Tabela 2 ).

Como não houve diferença significativa entre as formas de colocação das bolsas de decomposição sob SPC (Tabela 2), foi usada a média dos valores observados nessas duas formas de colocação para a análise de regressão pela qual se avaliou o efeito do tempo de amostragem na velocidade de decomposição das palhadas de crotalária e de sorgo.

Pela análise de regressão aos 60 DAC sob SPD, estima-se que ainda restavam cerca de $50 \%$ do material usado para a realização do teste; enquanto sob SPC a mesma quantidade remanescente (50\%) foi estimada aos $28 \mathrm{DAC}$ para o sorgo e aos 20 DAC para a crotalária (Figura 2A). Esses resultados corroboram com aqueles reportados por Dalal et al. (2007) em que, sob SPD, o tempo médio de permanência da palhada no solo foi mais do que o dobro em comparação com o SPC.

Portanto, sob SPD a velocidade de decomposição da palhada da crotalária e do sorgo não foi fortemente influenciada pelo tipo da planta (Figura 2A). Por outro lado, o maior contato entre as partículas de solo e os tecidos das plantas, causado pelo SPC, acelera o processo de decomposição principalmente para a palhada de crotalária, para a qual a literatura reporta menor relação $\mathrm{C} / \mathrm{N}$ em comparação com o sorgo (Silva et al., 2009).

Os resíduos vegetais contêm cerca de $40-50 \%$ de C em massa seca mas o seu teor de $\mathrm{N}$ varia consideravelmente, causando variação da razão $\mathrm{C} / \mathrm{N}$. Geralmente se acata que os resíduos com alta relação $\mathrm{C} / \mathrm{N}$ se decompõem mais lentamente do que aqueles com baixa relação C/N (Kumar \& Goh, 1999)

Considerando todo o período de avaliação (124 dias), a taxa de decomposição diária da palhada sob SPD foi de $615 \mathrm{mg}$ de

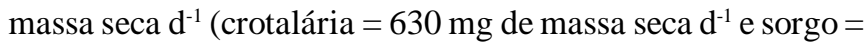

Tabela 2. Percentual de massa seca das plantas de cobertura do solo remanescente em função da forma de colocação das bolsas de decomposição nos sistemas plantio direto (SPD) e plantio convencional (SPC)

\begin{tabular}{lcccccc}
\hline \multirow{2}{*}{ Tratamento } & \multicolumn{5}{c}{ Época de amostragem (DAC) } \\
\cline { 2 - 7 } Crotalária/SPD/SS & $\mathbf{0}$ & $\mathbf{4}$ & $\mathbf{1 2}$ & $\mathbf{2 8}$ & $\mathbf{6 0}$ & $\mathbf{1 2 4}$ \\
Sorgo/SPD/SS & $100 \mathrm{Aa}$ & $86,65 \mathrm{Aab}$ & $77,05 \mathrm{ABbc}$ & $60,73 \mathrm{Acd}$ & $47,43 \mathrm{Ad}$ & $22,00 \mathrm{ABe}$ \\
Crotalária/SPC/FC & $100 \mathrm{Aa}$ & $89,30 \mathrm{Aab}$ & $81,00 \mathrm{Abc}$ & $65,00 \mathrm{Acd}$ & $52,95 \mathrm{Ad}$ & $25,40 \mathrm{Ae}$ \\
Sorgo/SPC/FC & $100 \mathrm{Aa}$ & $87,03 \mathrm{Aa}$ & $57,47 \mathrm{Cb}$ & $33,57 \mathrm{Bc}$ & $14,57 \mathrm{Bd}$ & $4,80 \mathrm{Cd}$ \\
Crotalária/SPC/Pe & $100 \mathrm{Aa}$ & $84,30 \mathrm{Aab}$ & $68,17 \mathrm{ABCb}$ & $49,27 \mathrm{ABc}$ & $27,07 \mathrm{Bd}$ & $9,83 \mathrm{ABCe}$ \\
Sorgo/SPC/Pe & $100 \mathrm{Aa}$ & $91,50 \mathrm{Aa}$ & $63,53 \mathrm{BCb}$ & $36,97 \mathrm{Bc}$ & $16,77 \mathrm{Bd}$ & $8,20 \mathrm{BCd}$ \\
\hline
\end{tabular}

Médias na coluna seguidas da mesma letra maiúscula e, na linha seguida pela mesma letra minúscula, não diferem entre si pelo teste de Tukey $(p<0,05)$

DAC - dias após a colocação das sacolas de decomposiçãa; SS - bolsas de decomposição deixadas na superfície do solo; FC - bolsas de decomposição colocadas no fundo da cova (20 cm); Pe - bolsas de decomposição colocadas ocupando o perfil do solo $(0-20 \mathrm{~cm})$ 


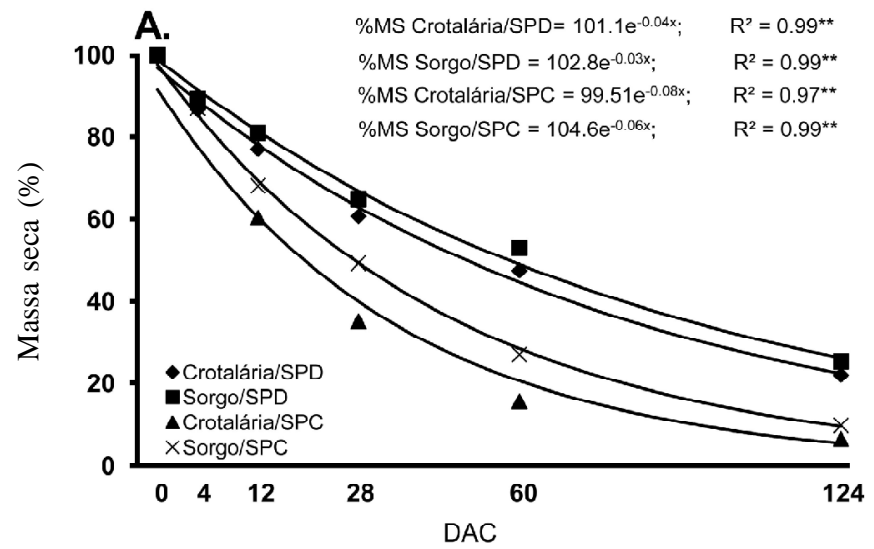

B.

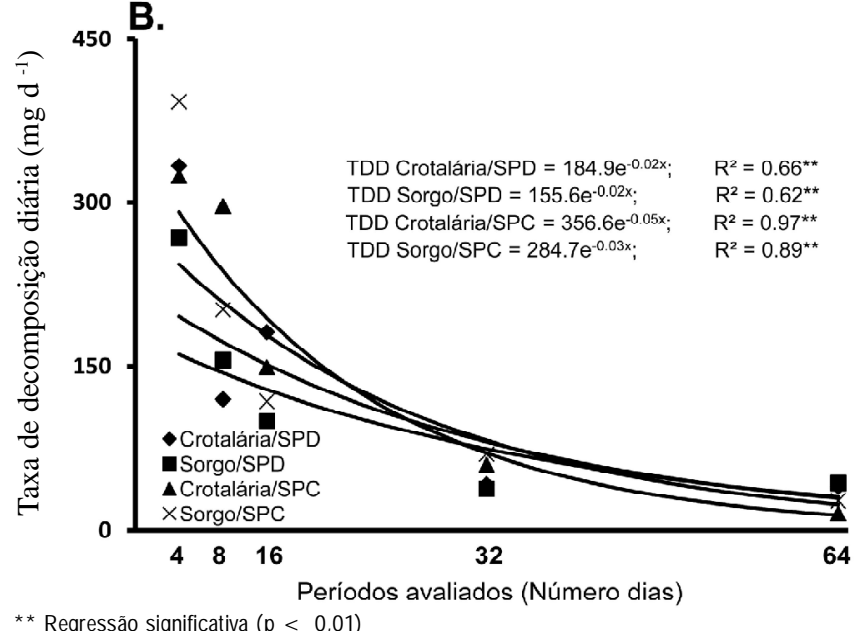

Figura 2. Decomposição de plantas de cobertura do solo (crotalária e sorgo) avaliada utilizando-se bolsas de decomposição. Percentual de massa seca remanescente nas bolsas de decomposição (A) e taxa de decomposição diária em cada período amostrado (B). DAC - Dias após a colocação das sacolas de decomposição; SPD - Sistema plantio direto; SPC - Sistema de plantio convencional

$600 \mathrm{mg}$ de massa seca $\left.\mathrm{d}^{-1}\right)$, enquanto sob SPC essa taxa foi de $735 \mathrm{mg}$ de massa seca $\mathrm{d}^{-1}$ (crotalária $=754 \mathrm{mg}$ de massa seca $\mathrm{d}^{-1}$ e sorgo $=716 \mathrm{mg}$ de massa seca $\left.\mathrm{d}^{-1}\right)$, confirmando que sob SPD a decomposição da palhada se dá de forma mais lenta (Dalal et al., 2007).

Além disso, durante grande parte do período de avaliação e se levando em conta os intervalos entre cada avaliação, tanto para crotalária quanto para sorgo, a taxa de decomposição foi bem mais lenta sob SPD (Figura 2B), significando que sob esse tipo de manejo do solo a liberação de nutrientes ocorre de forma mais uniforme durante o ciclo de culturas anuais, como o feijoeiro comum, o que permite melhor absorção dos nutrientes.

A produtividade de grãos da cultura do feijoeiro comum foi cerca de $40 \%$ maior sob SPD em relação ao SPC. Observou-se também que a produtividade do feijoeiro comum foi de $1.722 \mathrm{~kg}$ ha ${ }^{-1}$ após crotalária e $1.760 \mathrm{~kg} \mathrm{ha}^{-1}$ após sorgo, não se observando efeito significativo das culturas de cobertura do solo (Figura 3). Carvalho et al. (2007) também relataram maior produtividade da cultura do feijoeiro comum sob SPD e, apesar das plantas de cobertura terem influenciado no acúmulo de nutrientes, não interferiram na produtividade do feijoeiro.

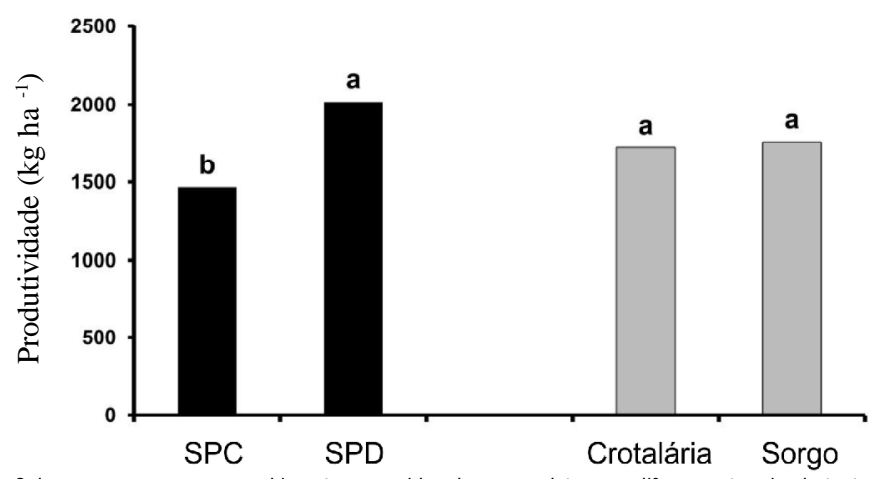

Colunas com mesmo preenchimento e seguidas da mesma letra não diferem entre si pelo teste de Tukey $(p<0,05)$

Figura 3. Produtividade de grãos da cultura do feijoeiro comum sob sistema plantio direto (SPD) e sistema de plantio convencional (SPC) e em razão da espécie da planta de cobertura do solo

Apesar de não se ter observado efeito das plantas de cobertura do solo na produtividade do feijoeiro comum, a longo prazo a associação dessas plantas com o sistema de manejo do solo poderá resultar em ganhos em produtividade das culturas, em função da melhoria das qualidades químicas, físicas e biológicas do solo (D’Andréa et al., 2002). Segundo Stone \& Silveira (1999), o preparo convencional do solo, em condições irrigadas proporcionou, ao feijoeiro, menor produtividade em relação ao sistema plantio direto. Além da maior disponibilidade de água, a maior produtividade no sistema plantio direto tem sido atribuída também ao acúmulo de nutrientes na camada superficial e à maior atividade biológica, condicionada pela maior umidade e menor temperatura do solo, com reflexos na solubilização, na liberação de nutrientes e na eficiência da absorção pelas plantas (Stone \& Silveira, 1999).

A quantidade de $\mathrm{N}$ acumulada pelas plantas de cobertura do solo apresentou correlação positiva e significativa com a produtividade de grãos da cultura do feijoeiro comum, o que não foi observado entre quantidade de $\mathrm{N}$ versus produtividade de massa seca das plantas de cobertura do solo (Tabela 3).

Tabela 3. Coeficientes de correlação de Pearson entre as variáveis analisadas. Produção de massa seca pelas plantas de cobertura do solo - PMS (Mg ha-1), Q uantidade de N fornecida pelas plantas de cobertura do solo - Q N (kg ha-1)e Produtividade de grãos do feijoeiro comum - PGF (kg ha-1)

\begin{tabular}{|c|c|c|c|}
\hline & PMS & QN & PGF \\
\hline PMS & 1 & $0.44^{\mathrm{ns}}$ & $0.34^{\text {ns }}$ \\
\hline QN & - & 1 & $0.99 * *$ \\
\hline PGF & - & - & 1 \\
\hline
\end{tabular}

A maior produtividade de grãos da cultura do feijoeiro comum sob SPD (Figura 3) pode estar diretamente relacionada à maior quantidade de $\mathrm{N}$ acumulada pelas plantas de cobertura do solo sob SPD (Figura 1B). Este resultado corrobora com os descritos por Souza et al. (2002), em que o rendimento máximo da cultura do feijoeiro comum foi obtido com quantidades de calcário e fertilizantes, incluindo-se os nitrogenados, correspondentes a 1,34 vez a dose de referência empregada. Apesar da resposta 
da cultura ao $\mathrm{N}$ depender de vários fatores, inclusive da cultivar utilizada, em geral doses de nitrogênio em cobertura promovem acréscimos lineares nas produtividades de grãos da cultivar Pérola, no sistema de plantio direto (Fornasieri Filho et al., 2007).

\section{ConclusõEs}

1. O sorgo produz mais massa seca que a crotalária; contudo o sistema de manejo do solo não influencia na produtividade de massa seca das plantas de cobertura.

2. O sistema plantio direto (SPD) possibilitou maior acúmulo de N que o sistema de plantio convencional (SPC); entretanto, as plantas de cobertura do solo acumularam a mesma quantidade de N.

3. A taxa de decomposição das plantas de cobertura do solo sob SPD foi cerca de $50 \%$ menor do que sob SPC e, até os 28 dias, a decomposição da crotalária sob SPC acontece de forma mais rápida que a do sorgo.

4. A maior produtividade de grãos da cultura do feijoeiro comum ocorre em SPD.

5. A produtividade de grãos do feijoeiro comum está altamente correlacionada à quantidade de $\mathrm{N}$ acumulada pelas plantas de cobertura do solo.

\section{Agradecimentos}

Os autores agradecem ao Convênio INCRA/FAPED/ EMBRAPA pelo apoio financeiro.

\section{LITERATURA CITADA}

Aguiar, R. A.; Moreira, J. A. A.; Stone, L. F.; Bernardes, T. G.; Jesus, R. P. Sustentabilidade de sistemas orgânicos com plantas de cobertura na cultura do arroz, por meio de alterações físicas do solo. Pesquisa Agropecuária Tropical, v.40, p.142-149, 2010.

Altieri, M. A. Sustainable agriculture. Encyclopedia of Agricultural Science, v.4, p.239-247, 1994.

Altieri, M. A. The ecological role of biodiversity in agroecosystems. Agriculture, Ecosystems and Environment, v.74, p.19-31, 1999.

Andrade, R. S.; Stone, L. F.; Silveira, P. M. Culturas de cobertura e qualidade física de um Latossolo em plantio direto. Revista Brasileira de Engenharia Agrícola e Ambiental, v.13, p.411418, 2009.

Barbosa Filho, M. P.; Silva, O. F. Adubação e calagem para o feijoeiro irrigado em solo de cerrado. Pesquisa Agropecuária Brasileira, v.35, p.1317-1324, 2000.

Bordin, L.; Farinelli, R.; Penariol, F. G.; Fornasieri Filho, D. Sucessão de cultivos de feijão-arroz com doses de adubação nitrogenada após adubação verde, em semeadura direta. Bragantia, v.62, p.417-428, 2003.
Carneiro, R. G.; Mendes, I. C.; Lovato, P. E.; Carvalho, A. M.; Vivaldi, L. J. Indicadores biológicos associados ao ciclo de fósforo em solos de Cerrado sob plantio direto e plantio convencional. Pesquisa Agropecuária Brasileira, v.39, p.661669, 2004.

Carvalho, M. A. C.; Athayde, L. F.; Soratto, R. P.; Alves, M. C.; Sá, M. E. Adubação verde e sistemas de manejo do solo na produtividade do algodoeiro. Pesquisa Agropecuária Brasileira, v.39, p.1205-1211, 2004.

Carvalho, M. A. C.; Soratto, R. P.; Alves, M. C.; Arf, O.; Sá, M. E. Plantas de cobertura, sucessão de culturas e manejo do solo em feijoeiro. Bragantia, v.66, p.659-668, 2007.

Castellini, C.; Bastianoni, S.; Granai, C.; Dal Bosco, A.; Brunetti, M. Sustainability of poultry production using the energy approach: Comparison of conventional and organic rearing systems. Agriculture, Ecosystems and Environment, v.114, p.343-350, 2006.

Chu, G. X.; Shen, Q. R.; Cao, J. L. Nitrogen fixation and N transfer from peanut to rice cultivated in aerobic soil in an intercropping system and its affect on soil $\mathrm{N}$ fertility. Plant and Soil, v.263, p.17-27, 2004.

Cunha, E. Q.; Stone, L. F.; Moreira, J. A. A.; Ferreira, E. P. B.; Didonet, A. D. Atributos físicos do solo sob diferentes preparos e coberturas influenciados pela distribuição de poros. Revista Brasileira de Engenharia Agrícola e Ambiental, v.14, p.1160-1169, 2010.

D’Andréa, A. F.; Silva, M. L. N.; Curi, N.; Ferreira, M. M. Atributos biológicos indicadores da qualidade do solo em sistemas de manejo na região do cerrado no sul do Estado de Goiás. Revista Brasileira de Ciência do Solo, v.26, p.913-923, 2002.

Dalal, R. C.; Strong, W. M.; Cooper, J. E.; King, A. J. No-tillage and nitrogen application affects the decomposition of ${ }^{15} \mathrm{~N}$ labelled wheat straw and the levels of mineral nitrogen and organic carbon in a Vertisol. Australian Journal of Experimental Agriculture, v.47, p.862-868, 2007.

Faria, S. M.; Franco, A. A. Identificação de bactérias eficientes na fixação biológica de nitrogênio para espécies leguminosas arbóreas. Seropédica: Embrapa Agrobiologia, 160. 2002. Documento, 158

Ferreira, D. F. Sisvar 4.3. 2003. http://www.dex.ufla.br/danielff/ sisvar. Acessado em 16 de agosto de 2010.

Ferreira, E. P. B.; Santos, H. P.; Costa, J. R.; De-Polli, H.; Rumjanek, N. G. Microbial soil quality indicators under different crop rotations and tillage management. Revista Ciência Agronômica, v.41, p.177-183, 2010.

Fornasieri Filho, D.; Xavier, M. A.; Lemos, L. B.; Farinelli, R. Resposta de cultivares de feijoeiro comum à adubação nitrogenada em sistema de plantio direto. Científica, v.35, p.115-121, 2007.

Kaltsas, A. M.; Mamolos, A. P.; Tsatsarelis, C. A.; Nanos, G. D.; Kalburtji, K. L. Energy budget in organic and conventional olive groves. Agriculture, Ecosystems and Environment, v.122, p.243-251, 2007.

Kumar, K.; Goh, K. M. Crop residues and management practices: effects on soil quality, soil nitrogen dynamics, crop yield, and nitrogen recovery. Advances in Agronomy, v.68, p.197$319,1999$. 
Landers, J. N. Zero tillage development in tropical Brazil - the story of a successful NGO activity. Rome: FAO, 2001. 69p. FAO. Agricultural Services Bulletin, 147.

Paula Júnior, T. J.; Vieira, R. F.; Teixeira, H.; Coelho, R. R.; Carneiro, J. E. S.; Andrade, M. J. B.; Resende, A. M. Informações técnicas para o cultivo de feijoeiro-comum na região central brasileira: 2007-2009. Viçosa: EPAMIG, 2008. 180p.

Pietsch, G.; Friedel, J. K.; Freyer, B. Lucerne management in an organic farming system under dry site conditions. Field Crops Research, v.102, p.104-118, 2007.

Roldán, A.; Caravaca, F.; Hernández, M.T.; García, C.; SánchesBrito, C.; Velásquez, M.; Tiscareño, M. No-tillage, crop residue additions, and legume cover cropping effects on soil quality characteristics under maize in Patzcuaro wathershed (Mexixo). Soil and Tillage Research, v.72, p.6573, 2003.

Roldán, A.; Salinas-García, J. R.; Alguacil, M. M.; Caravaca, F. Soil sustainability indicators following conservation tillage practices under subtropical maize and bean crops. Soil and Tillage Research, v.93, p.273-282, 2007.

Roscoe, R.; Boddey, R. M.; Salton, J. C. Sistema de manejo e matéria orgânica do solo. In: Roscoe, R.; Mercante, F. M.; Salton, J. C. Dinâmica da matéria orgânica do solo em sistemas conservacionistas: modelagem matemática e métodos auxiliares. Dourados: Embrapa Agropecuária Oeste, 2006. p.17-42.

Santos, A. B. dos; Fageria, N. K.; Silva, O. F. da; Melo, M. L. B. Resposta do feijoeiro ao manejo de nitrogênio em várzeas tropicais. Pesquisa Agropecuária Brasileira, v.38, p.1265-1271, 2003.

Silva, C. C; Del Peloso, M. J. Informações técnicas para o cultivo do feijoeiro comum na Região Central-brasileira 2005-2007. Santo Antônio de Goiás: Embrapa Arroz e Feijão, 2006. 139p. Documentos, 193.
Silva, P. C. G.; Foloni, J. S. S.; Fabris, L. B.; Tiritan, C. S. Fitomassa e relação $\mathrm{C} / \mathrm{C}$ em consórcios de sorgo e milho com espécies de cobertura. Pesquisa Agropecuária Brasileira, v.44, p.1504$1512,2009$.

Silva, S. C.; Xavier, L. S.; Santana, N. M. P.; Cardoso, G. M.; Pelegrini, J. C. Informações meteorológicas para pesquisa e planejamento agrícola referente ao município de Santo Antônio de Goiás, GO. Santo Antônio de Goiás: Embrapa Arroz e Feijão, 21p. 2002. Documento, 136

Souza, A. B.; Andrade, M. J. B.; Muniz, J. A.; Reis, R. P. Populações de plantas e níveis de adubação e calagem para o feijoeiro (Phaseolus vulgaris L.) em um solo de baixa fertilidade. Ciência e Agrotecnologia, v.26, p.87-98, 2002.

Stone, L. F.; Silveira, P. M. da. Efeitos do sistema de preparo na compactação do solo, disponibilidade hídrica e comportamento do feijoeiro. Pesquisa Agropecuária Brasileira, v.34, p.83-91, 1999.

Tedesco, M. J.; Gianello, C.; Bissani, C. A.; Bohnen, H.; Volkweiss, S. J. Análises de solo, plantas e outros materiais. 2.ed. Porto Alegre: Universidade Federal do Rio Grande do Sul, 1995. 174p.

Teixeira, F. C. P.; Reinert, F.; Rumjanek, N. G.; Boddey, R. M. Quantification of the contribution of biological nitrogen fixation to Cratylia mollis using the ${ }^{15} \mathrm{~N}$ natural abundance technique in the semi-arid caatinga region of Brazil. Soil Biology \& Biochemistry, v.38, p.1989-1993, 2006.

Waksman, S. A.; Tenney, F. G.; Stevens, K. R. The role of microorganisms in the transformation of organic matter in forest soils. Ecology, v.9, p.126-144, 1928.

Welch, R. M.; Graham, R. D. A new paradigm for world agriculture: meeting human needs productive, sustainable, nutritious. Field Crops Research, v.60, p.1-10, 1999.

Wessa P. Pearson Correlation (v1.0.3) in Free Statistics Software (v1.1.23-r4), Office for Research Development and Education, 2008. http://www.wessa.net/rwasp_correlation.wasp/. 10 Ago. 2010. 